Bundesgesundheitsbl 2015 · 58:758

DOI 10.1007/s00103-015-2179-5

Online publiziert: 19. Mai 2015

c) Springer-Verlag Berlin Heidelberg 2015

\section{Monika Mund}

Dezernat 7, Kassenärztliche Bundesvereinigung, Berlin, Deutschland

Kommentar zum

Beitrag von Linder et al:

Disease-Management-

Programme bei der

Nutzenbewertung.

Bundesgesundheitsbl

Gesundheitsforsch

Gesundheitsschutz 2015: Heft 4/5

Zu dem Artikel Linder et al.: DiseaseManagement-Programme. Schwierigkeiten bei der Nutzenbewertung. Bundesgesundheitsbl, Gesundheitsforsch, Gesundheitsschutz 2015, Heft 4/5 bitten wir folgenden Kommentar zu veröffentlichen:

Die Berechnung der Gesamtkosten für Disease-Management-Programme ist fehlerhaft, da die DMP-Einschreibungen nicht um Mehrfachteilnahme bereinigt wurden. Korrekt wäre die Verwendung der RSA-wirksam eingeschriebenen Versicherten. Es resultieren daher erhebliche Überschätzungen bei der Darstellung des finanziellen Aufwandes für DMP im gesamten Artikel. Transparent wäre zusätzlich ein Hinweis auf die Überschätzung durch Nichtberücksichtigung von unterjährig Versicherten. Die im Artikel zitierte Quelle der KBV (40) enthält keine Informationen, die als Basis für die dargestellten Berechnungen dienen könnten, es werden dort ausschließlich Daten zum DMP Diabetes Typ 2 dargestellt.

\section{Korrespondenzadresse}

\section{Dr. M. Mund}

Dezernat 7

Kassenärztliche Bundesvereinigung, Herbert-

Lewin-Platz 2, 10623 Berlin

MMund@kbv.de 